\title{
Recent results on the meson and baryon spectrum from lattice QCD
}

\author{
Daniel Mohler ${ }^{1,2, a}$ \\ ${ }^{1}$ Helmholtz-Institut Mainz, 55099 Mainz, Germany \\ 2 Johannes Gutenberg Universität Mainz, 55099 Mainz, Germany
}

\begin{abstract}
Recent lattice results on the meson and baryon spectrum with a focus on the determination of hadronic resonance masses and widths using a combined basis of singlehadron and hadron-hadron interpolating fields are reviewed. These mostly exploratory calculations differ from traditional lattice QCD spectrum calculations for states stable under QCD, where calculations with a full uncertainty estimate are already routinely performed. Progress and challenges in these calculations are highlighted.
\end{abstract}

\section{Introduction}

In recent years, tremendous progress has been made in calculating QCD observables from first principles using Lattice QCD. In particular, it is now possible to perform calculations at physical light-quark masses and with multiple lattice spacings and volumes. Improved actions have been developed for simulations with both light $(\mathrm{u}, \mathrm{d}, \mathrm{s})$ and heavy $(\mathrm{c}, \mathrm{b})$ quarks, allowing for a reliable extraction of simple observables for mesons made from these quarks.

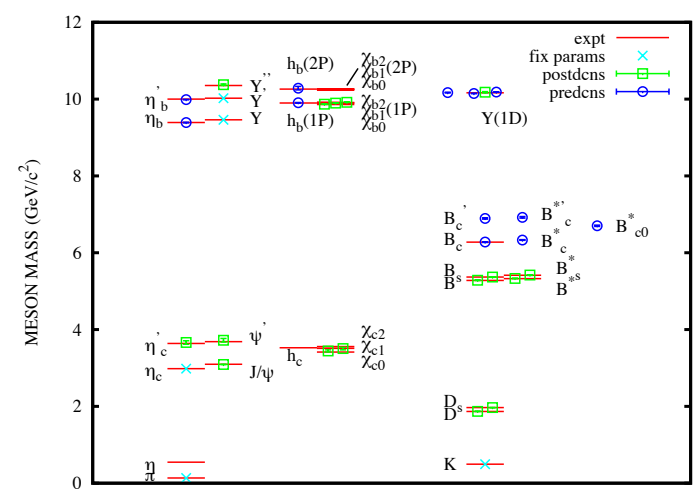

Figure 1. The spectrum of mesons comparing HPQCD lattice QCD results to experiment. Crosses are quantities used to fix parameters in the action, while the other results were either postdictions or predictions. Figure from [1].

Figure 1 (from [1]) shows results for the lattice spectrum of states stable or nearly stable under the strong interaction compared to experiment. After some of the experimental masses are used as input to tune the mass parameters and coupling in the action (cyan crosses in the figure), parameter free theory postdictions and predictions were obtained.

\footnotetext{
ae-mail: mohler@kph.uni-mainz.de
} 


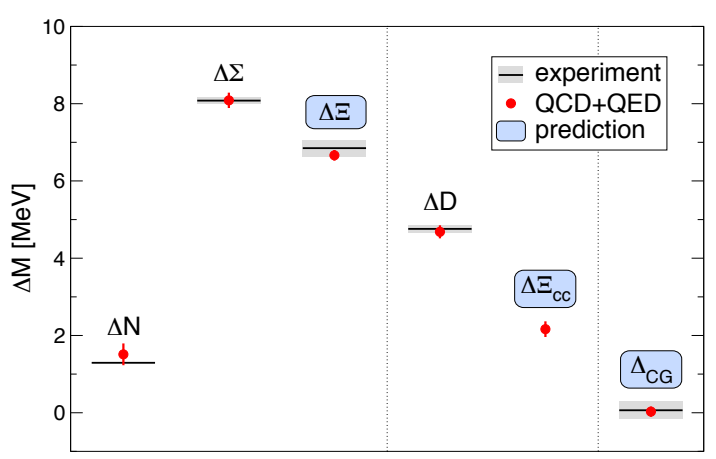

Figure 2. Mass splittings in channels stable under the strong and electromagnetic interactions. The red dots and uncertainties are the lattice data and the lines and shaded bands are the experimental results. Figure from [2].

Beyond pure QCD, recent progress has allowed the dynamical simulation of both QCD and QED on the lattice. Figure 2 (from [2]) shows the isospin splitting of various ground state hadrons, resulting from both the difference of up and down quark masses and from QED. The resulting mass splittings agree perfectly with those measured in experiment, while splittings not yet observed to date in experiment can also be calculated.

In these proceedings we address more challenging observables, taking a look at hadrons close to multiparticle thresholds and hadronic resonances. While the masses of stable states well separated from multi-particle thresholds are readily extracted in modern lattice calculations, extracting the spectrum of hadronic excitations is a challenge. In Section 2.1 readers will be pointed to literature relevant to understand theoretical aspects of extracting scattering observables from Lattice QCD. In the remainder of Section 2 the current state of the art with regard to both meson-meson and mesonbaryon scattering are briefly reviewed by appealing to specific examples from the literature. In Section 2.2 examples for meson-meson scattering studies will be presented, while 2.3 highlights some of the findings from these studies. In Section 2.4 progress in the simulation of meson-baryon scattering is discussed. Section 3 provides a brief outlook.

\section{Spectroscopy of resonances and close-to-threshold states}

\subsection{Lüscher's finite volume method}

From Euclidean space correlation functions, scattering observables are not accessible directly. In a series of seminal papers by Lüscher [3-5] it was pointed out that information about the continuum scattering amplitude of elastic scattering can be inferred from the finite volume dependence of the energy levels, which appear shifted from the free energy levels due to the interaction. These energy shifts are illustrated in Figure 3, where the energies for the scattering of two bosons in a channel with a resonance at three times the boson mass are drawn as a function of the box size.

The relations first provided by Lüscher have been greatly generalized over the years, now including relations for any number of (coupled) two particle channels, for $2 \leftrightarrow 1$ and $2 \leftrightarrow 2$ transitions (such as $\pi \pi \rightarrow \pi \gamma^{*}$ ), for particles with and without spin, for moving frames, etc.. For recent reviews of the formalism including the relevant references please refer to [6,7]. In practical applications it is crucial to be able to obtain enough lattice energy levels in the region of interest. To this end both multiple volumes and multiple momentum frames are used. 


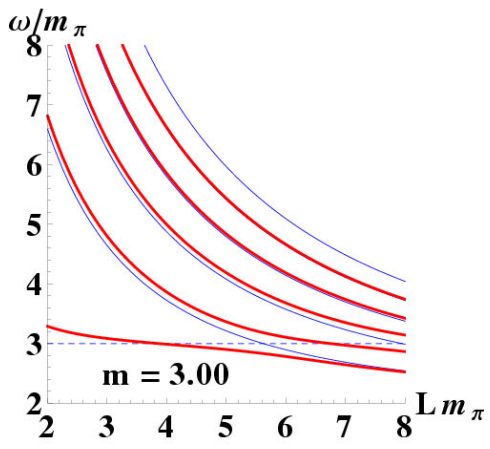

Figure 3. Energies for the scattering of two bosons in a channel with a resonance at three times the boson mass as a function of the box size. The blue lines represent the non-interacting spectrum, while the red lines displaying a characteristic avoided level crossing pattern show the expectation in presence of the interaction.

\subsection{Meson-meson scattering and resonances}

\subsubsection{The $\rho$ meson}

The simplest QCD resonance is the $\rho$ meson with quantum numbers $J^{P C}=1^{--}$, seen in isospin-1 $\pi \pi$ scattering. It decays nearly $100 \%$ of the time into two pions [8] and there seem to be no significant inelastic contributions from the open four pion threshold. For these reasons the $\rho$ meson is the ideal benchmark for lattice studies of elastic scattering employing the Lüscher formalism. After a pioneering study by the CP-PACS collaboration [9], multiple groups provided proof of principle calculations demonstrating the feasibility of lattice resonance studies [10-12]. In the meantime a number of further studies exist [13-18].

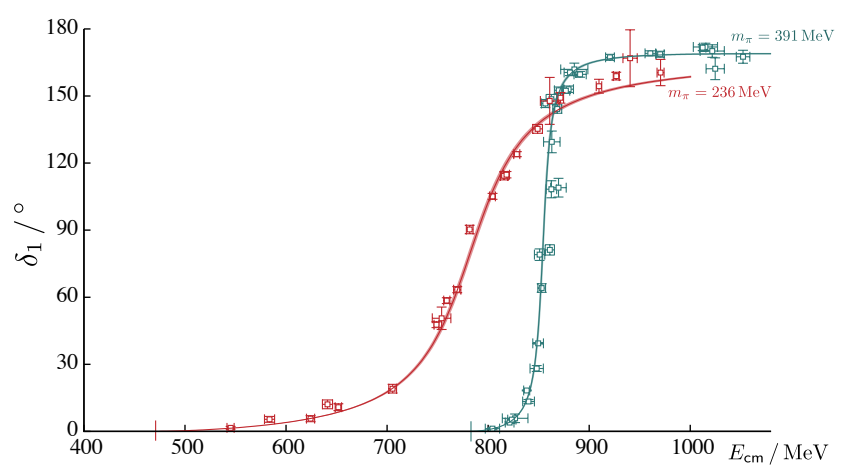

Figure 4. Elastic P-wave $\pi \pi$ scattering phase shifts with isospin 1 for two different pion masses. The blue data points are for $M_{\pi}=391 \mathrm{MeV}$ and the red data points for $M_{\pi}=236 \mathrm{MeV}$. Figure from [15].

Figure 4 shows an example of a state-of-the-art calculation by the Hadron Spectrum Collaboration [15]. The figure shows results for the p-wave phase shift $\delta_{1}$ of elastic $\pi \pi$ scattering for two unphysically large pion masses as a function of the center of mass energy $E_{c m}$. Using a Breit-Wigner parameterization, the resonance mass and coupling $g_{\rho \pi \pi}$ can be extracted. The large number of lattice data points results from using all lattice irreps for multiple center-of-mass momenta, and multiple lattice volumes.

Figure 5 shows the current world data for $g_{\rho \pi \pi}$ from the lattice determinations [10-18]. While there is in general a good agreement among the lattice data, the uncertainties in the plot are (in most cases) statistical only and there is no calculation demonstrating a full control of systematic uncertainties. 

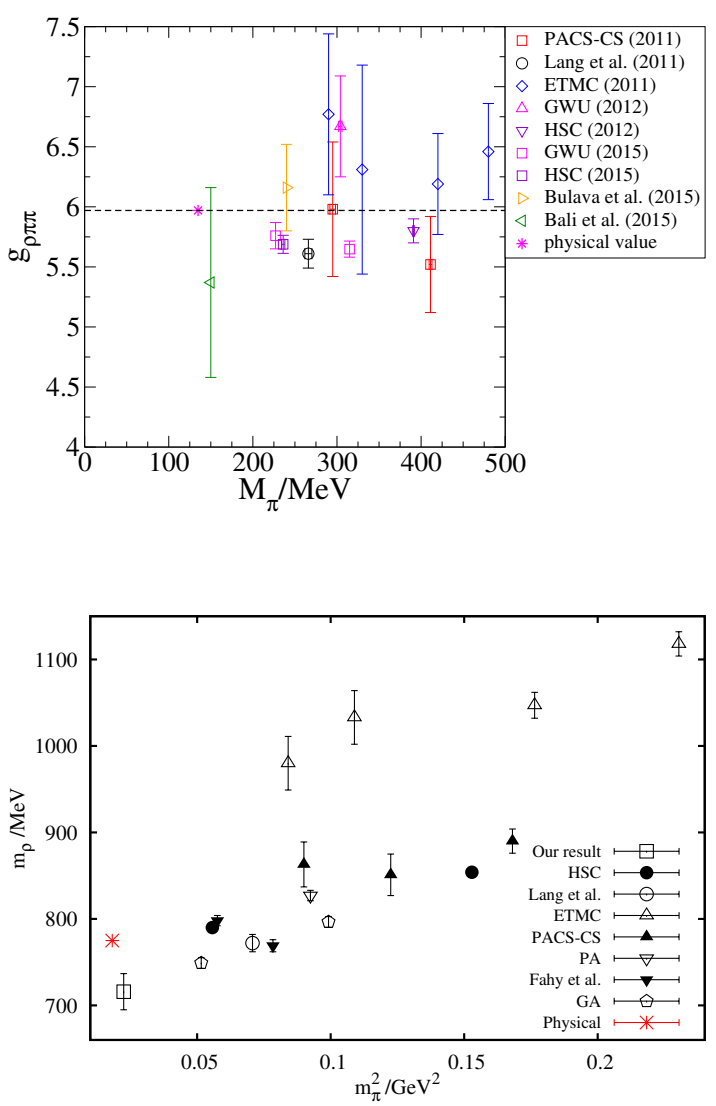

Figure 5. Collection of results from various lattice determination of the coupling $g_{\rho \pi \pi}$. For a list of references please refer to the text.

Figure 6. Collection of results from various lattice determination of the $\rho$ meson resonance mass taken from [16]. Notice that deviations are expected at non-physical pion masses as different groups use different ways to determine the physical quark masses and to set the scale in their lattice calculations.

A similar plot for the extracted resonance masses is shown in Figure 6, taken from [16]. It illustrates that the neglected systematics are likely important, as the resonance masses from different simulations tend to deviate quite a bit from each other at unphysical pion masses. Notice however that some deviations are expected as different groups use different ways to determine the physical quark masses and to set the scale in their lattice calculations. As a dimensionful quantity, the resonance mass therefore should only agree for physical parameters and in the continuum limit. Demonstrating this agreement will be a task for future lattice simulations.

With the growing dataset for the $\rho$-resonance the question naturally arises if there are additional insights gained from taking a more detailed look at the current set of results. In [19] the authors used Unitarized Chiral Perturbation Theory (Unitarized $\chi \mathrm{PT}$ ) to analyze the lattice scattering data with 2 flavors of light (up and down) dynamical quarks. Fitting to experiment data in addition, the Unitarized $\chi \mathrm{PT}$ calculation allows to "switch on" the strange quarks. As their final result the authors claim that the low resonance masses observed in 2 flavor calculations (compared to the physical rho and the corresponding $2+1$ flavor calculations) are a result of the missing $\bar{K} K$ channel. If confirmed this constitutes a striking effect from the (partial) quenching of the strange quark. Future lattice calculations with both 2 and $2+1$ flavors of dynamical quarks will shed light on this issue.

Beyond the resonance mass and couplings, a lattice determination of the phase shift $\delta_{1}$ and its derivative also allows for the determination of the pion timelike form factor in the elastic region 

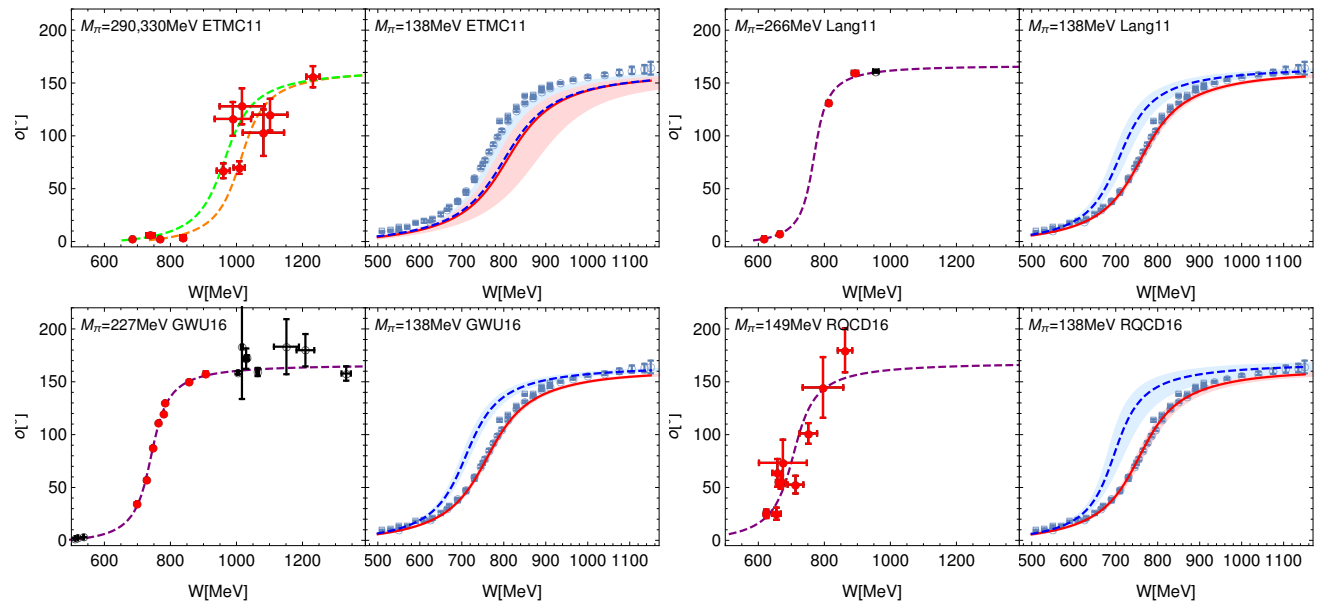

Figure 7. The left side in each plot shows lattice results from 2-flavor simulations of the $\rho$ meson from a subset of results analyzed in [19]. The right side in each plot shows the chirally extrapolated 2-flavor data (blue dashed curves and blue error bands) and the results after including the $\bar{K} K$ channel (red curves and bands) compared to the experimental data (blue circles and squares). Plot from [19].

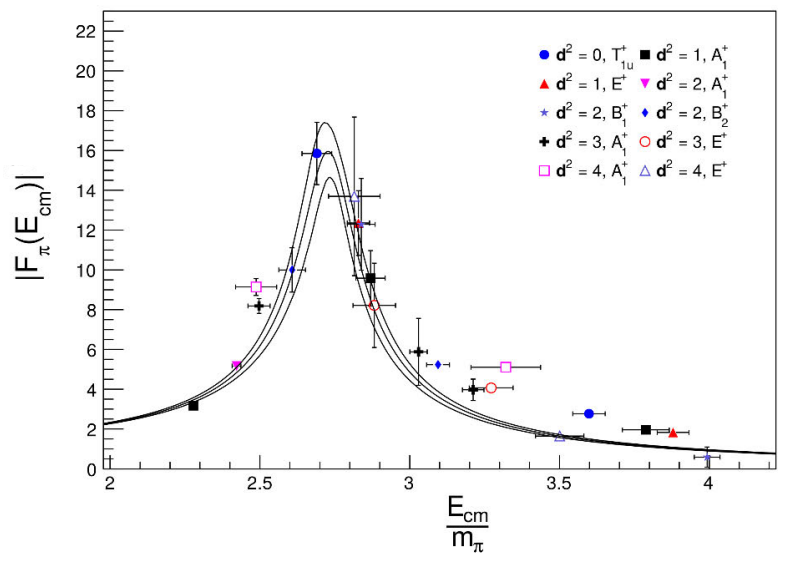

Figure 8. $\left|F_{\pi}(\sqrt{(s)})\right|$ as extracted from a lattice QCD calculation at $m_{\pi}=240 \mathrm{MeV}$ in [20]. The curve corresponds to a Gounaris-Sakurai parameterization with $m_{\rho}$ and $g_{\rho \pi \pi}$ determined from the lattice data.

$2 m_{\pi} \leq \sqrt{s} \leq 4 m_{\pi}[21]$. In this region the form factor $F_{\pi}(E)$ is given by

$$
R(s)=\frac{1}{4}\left(1-\frac{4 m_{\pi}^{2}}{s}\right)^{\frac{3}{2}}\left|F_{\pi}(\sqrt{s})\right|^{2}=\frac{\sigma\left(e^{+} e^{-} \rightarrow \text { hadrons }\right)}{4 \pi \alpha(s)^{2} /(3 s)} .
$$

It is of phenomenological importance for lattice determinations of the hadronic vacuum polarization (HVP) contribution to $(g-2)_{\mu}$ [22]. Recently first lattice calculations of the form factor have been performed in [20, 23]. Figure 8 shows results at unphysical parameters from [20], compared to a Gounaris-Sakurai parameterization (not a fit). The displayed results are from a single $2+1$ flavor ensemble with $m_{\pi} \approx 280$. These recent calculations demonstrate that the timelike pion form factor can be extracted with a reasonable precision. 


\subsection{2 $D_{s 0}^{*}(2317)$ and $D_{s 1}(2460)$ and their b-quark cousins}

In the spectrum of positive parity $D_{s}$ mesons, the $J^{P}=0^{+} D_{s 0}^{*}(2317)$ and $1^{+} D_{s 1}(2460)$ have masses and properties not expected from potential models for $\bar{q} q$ mesons. A particularly peculiar fact is that their mass is essentially degenerate with the corresponding $D$ mesons, even though the strange quark is much heavier than the light up and down quarks. This fact lead to speculations that these states have an exotic structure, such as a tetraquark or molecular structure.

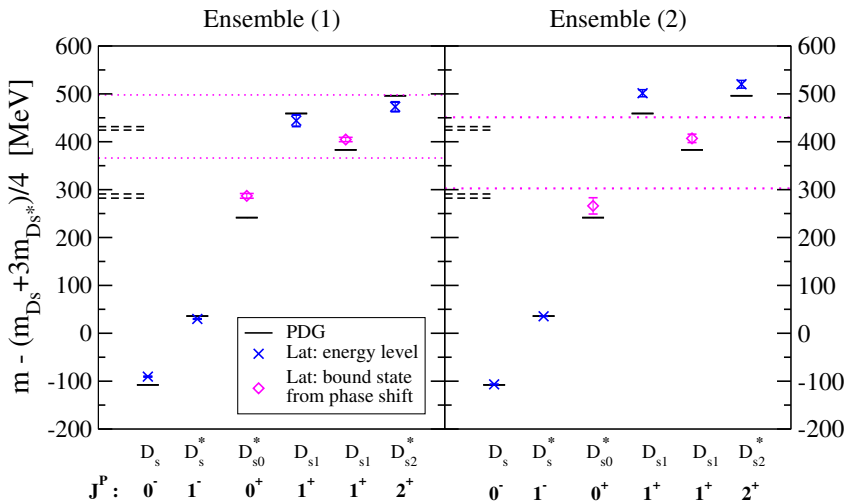

Figure 9. Low-lying $D_{s}$ meson spectrum from $[24,25]$ presented with respect to spin-averaged mass $\frac{1}{4}\left(m_{D_{s}}+3 m_{D_{s}^{*}}\right)$. The diamonds and crosses display our lattice results, while black full lines correspond to experiment. The magenta diamonds correspond to the pole position in the $T$-matrix. Masses extracted as energy levels in a finite box are displayed as blue crosses. Dotted (dashed) lines correspond to $D K$ and $D^{*} K$ lattice (physical) thresholds.

While traditional lattice studies using just quark-antiquark interpolating fields tend to get too large or badly determined masses for those states, a more recent study [24, 25] using both quark-antiquark and $D^{(*)} K$ structures leads to a spectrum in qualitative agreement with experiment, highlighting the role of the nearby thresholds. The results from this study are shown in Figure 9.
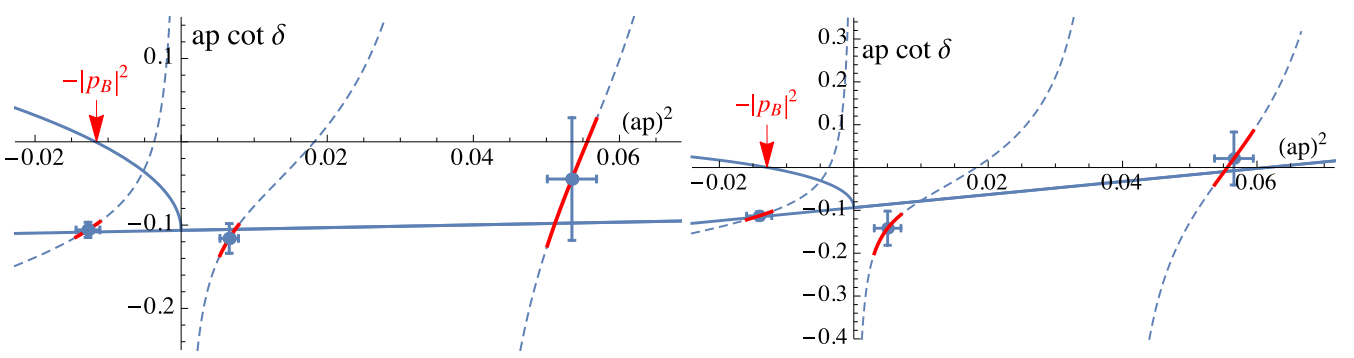

Figure 10. Plots of $a p \cot \delta(p)$ vs. $(a p)^{2}$ for $B^{(*)} K$ scattering in $s$-wave from [26]. Circles are values from our simulation; red lines indicate the error band following the Lüscher curves (dashed lines). The solid line gives the effective range fit to the points. The values for $-\left|p_{B}\right|^{2}$ corresponding to the binding energy in infinite volume are indicated by the arrows. Displayed uncertainties are statistical only.

While the LHCb experiment should be able to observe them, the corresponding $0^{+}$and $1^{+}$states in the $B_{s}$ spectrum are not yet observed in experiment. Using the finite volume formalism described in Section 2.1 and performing an effective range approximation close to threshold, a recent study [26] observed two bound states below the $B K$ and $B^{*} K$ thresholds (respectively). The lattice data along with the effective range parameterization is plotted in Figure 10. Figure 11 shows the resulting spectrum for the $1 \mathrm{~S}$ and $1 \mathrm{P}$ states. Notice that the known $1^{+}$and $2^{+}$mesons above threshold [8] are well reproduced. For more details please refer to [26]. 


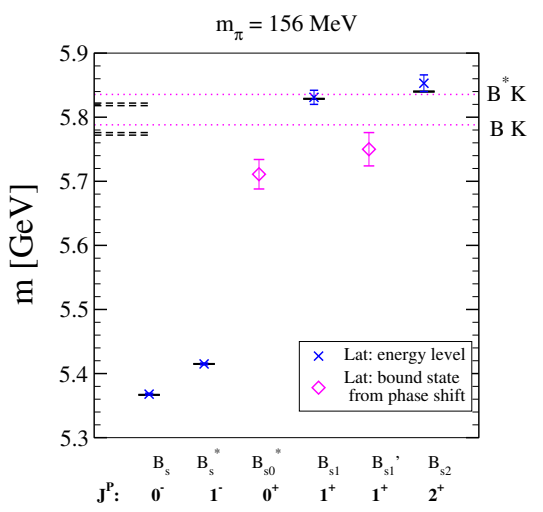

Figure 11. Spectrum of s-wave and p-wave $B_{s}$ states from [26]. The blue states are naive energy levels, while the bound state energy of the states in magenta results from an effective range approximation of the phase shift data close to threshold. The black lines are the energy levels from the PDG [8]. The error bars on the blue states are statistical only, while the errors on the magenta states show the full (statistical plus systematic) uncertainties.

\subsubsection{Coupled channel scattering and light scalar mesons}

Using 3 lattice volumes and several moving frames, the Hadron Spectrum Collaboration has started perform the first coupled-channel analyses of meson-meson systems in a finite volume. So far they have investigated coupled $\pi K-\eta K$ scattering [27, 28], $\pi \eta-K \bar{K}$ scattering [29], and extended their earlier $\rho$ meson study by also considering coupled channel $\pi \pi-K \bar{K}$ scattering [15].
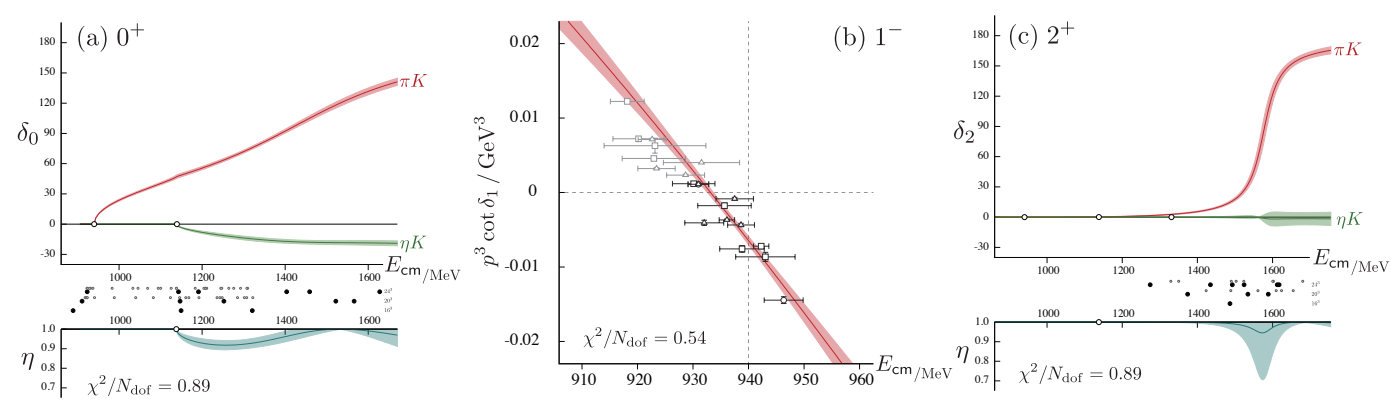

Figure 12. Results for $\pi K$ and $\eta K$ phase shifts with quantum numbers $J^{P}=0^{+}$(a), $1^{+}$(b), and $2^{+}$(c). For the coupled channel scattering with quantum numbers $0^{+}$and $2^{+}$the inelasticity $\eta$ is also plotted. Plot from [27]

Figure 12 shows their results for coupled channel $\pi K-\eta K$ scattering with $J^{P}=0^{+}, 1^{-}$and $2^{+}$. The low-lying physical resonances [8] in these channels are the $K_{0}^{*}(800)$ (also called $\kappa$ ) and the $K_{0}^{*}(1430)$ for $J^{P}=0^{+}$, the $K^{*}(892)$ for $J^{P}=1^{-}$, and the $K_{2}^{*}(1430)$ for $J^{P}=2^{+}$. The lattice results at an larger than physical pion mass of $391 \mathrm{MeV}$ qualitatively agree with the physical spectrum. In the $0^{+}$ channel shown in the left panel of Figure 12, a virtual bound state related to the physical $\kappa$ resonance is found along a much heavier and very broad $K_{0}^{*}$ resonance. The broad resonance is qualitatively compatible with the $K_{0}^{*}(1430)$. At such a large pion mass the $K^{*}(892)$ is not a resonance but a closeto-threshold bound state, and the corresponding coupling is compatible with the coupling extracted from experiment. The energy levels from which this bound state can be determined are depicted in the mid pane of the figure. An earlier single-channel lattice calculation [30,31] at a lighter pion mass of $266 \mathrm{MeV}$ already observed a very narrow resonance [31], also compatible with the $K^{*}(892)$. The right panel of Figure 12 shows the lattice data for the $J^{P}=2^{+}$channel, where a narrow resonance 


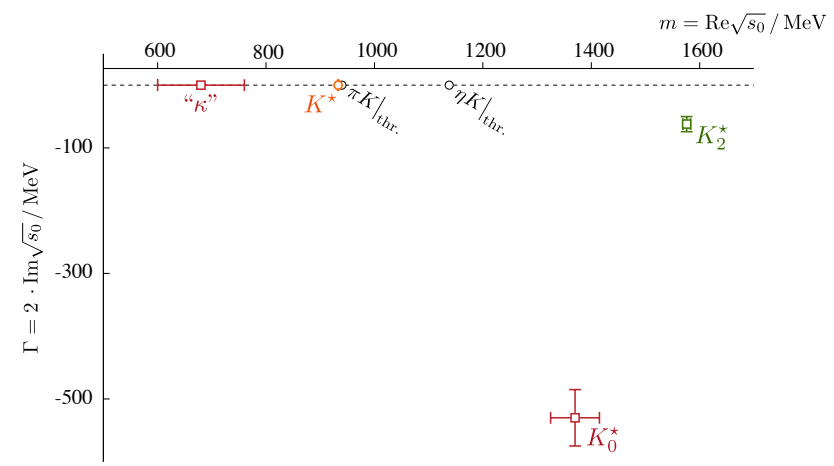

Figure 13. Pole positions in the complex plain for states with quantum numbers $0^{+}$ (red) $1^{-}$(orange) and $2^{+}$(green). For a full description please refer to $[27,28]$.

compatible with the $K_{2}^{*}(1430)$ is observed. Above the $\eta K$ threshold the inelasticity $\eta$ shown for $J^{P}=$ $0^{+}$and $1^{+}$remains large, indicating that these channels are coupled weakly. Figure 13 summarizes the findings, showing the complex poles determined from the lattice data. For more details on the analysis please refer to $[27,28]$.
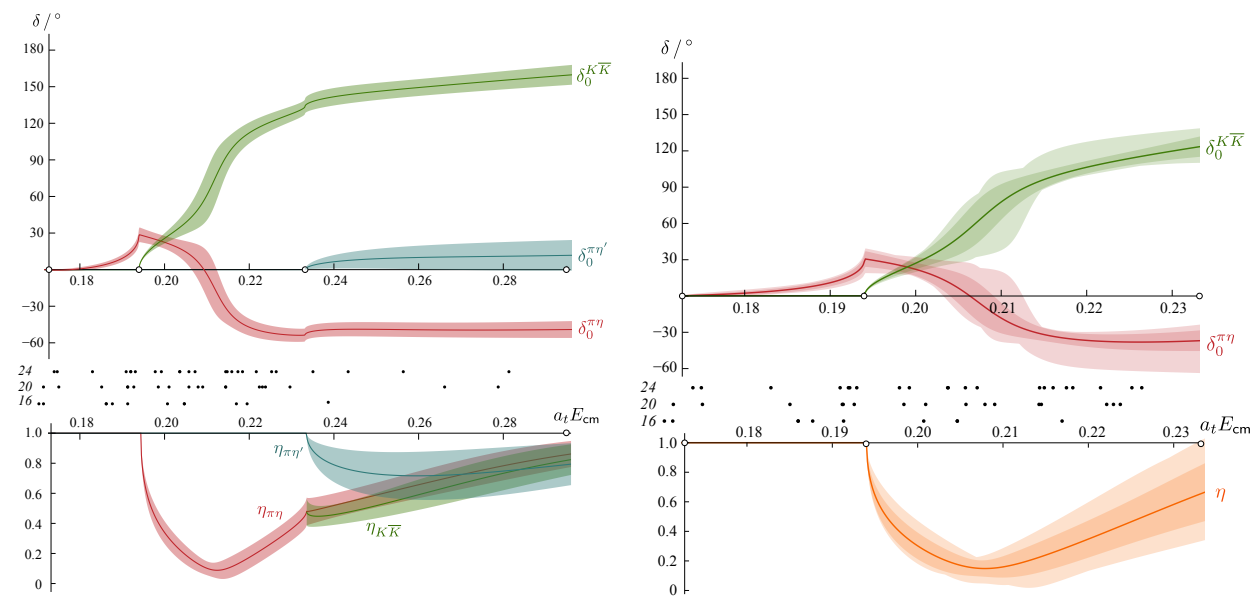

Figure 14. Left pane: The S-wave phase shift and inelasticities with three coupled channels $(\pi \eta, K \bar{K})$ and $\pi \eta^{\prime}$ in the isovector, scalar $\left(I\left(J^{P}\right)=1\left(0^{+}\right)\right)$channel. Right pane: Both plots from [29].

An example for a calculation involving tightly coupled channels is given by $\pi \eta-K \bar{K}$ scattering [29] with quantum numbers $I\left(J^{P}\right)=1\left(0^{+}\right)$. Here up to three coupled channels (also including $\pi \eta^{\prime}$ ) were considered. The lhs panel of Figure 14 shows the corresponding results for the phase shifts and inelasticities at a pion mass of $391 \mathrm{MeV}$. The rhs panel illustrates the systematic uncertainties from varying the parameterizations used below the $\pi \eta^{\prime}$ threshold. All successful parameterizations lead to a pole close to the $K \bar{K}$ threshold on a single unphysical Riemann sheet. This pole is likely related to the physical $a_{0}(980)$. For a full discussion of the results the reader is referred to [29]. In addition the lattice data for the $\mathrm{D}$-wave scattering features a narrow resonance, possibly related to the physical $a_{2}(1320)$. 


\subsection{A lesson from meson-meson scattering}

Studies of meson-meson scattering have demonstrated that a diverse basis of lattice interpolating operators is needed to reliably extract the finite volume spectrum. For hadron-hadron scattering this means in particular that to ensure good overlap with the physical states, all relevant meson-meson or meson-baryon interpolators should be included explicitly in the correlator basis.
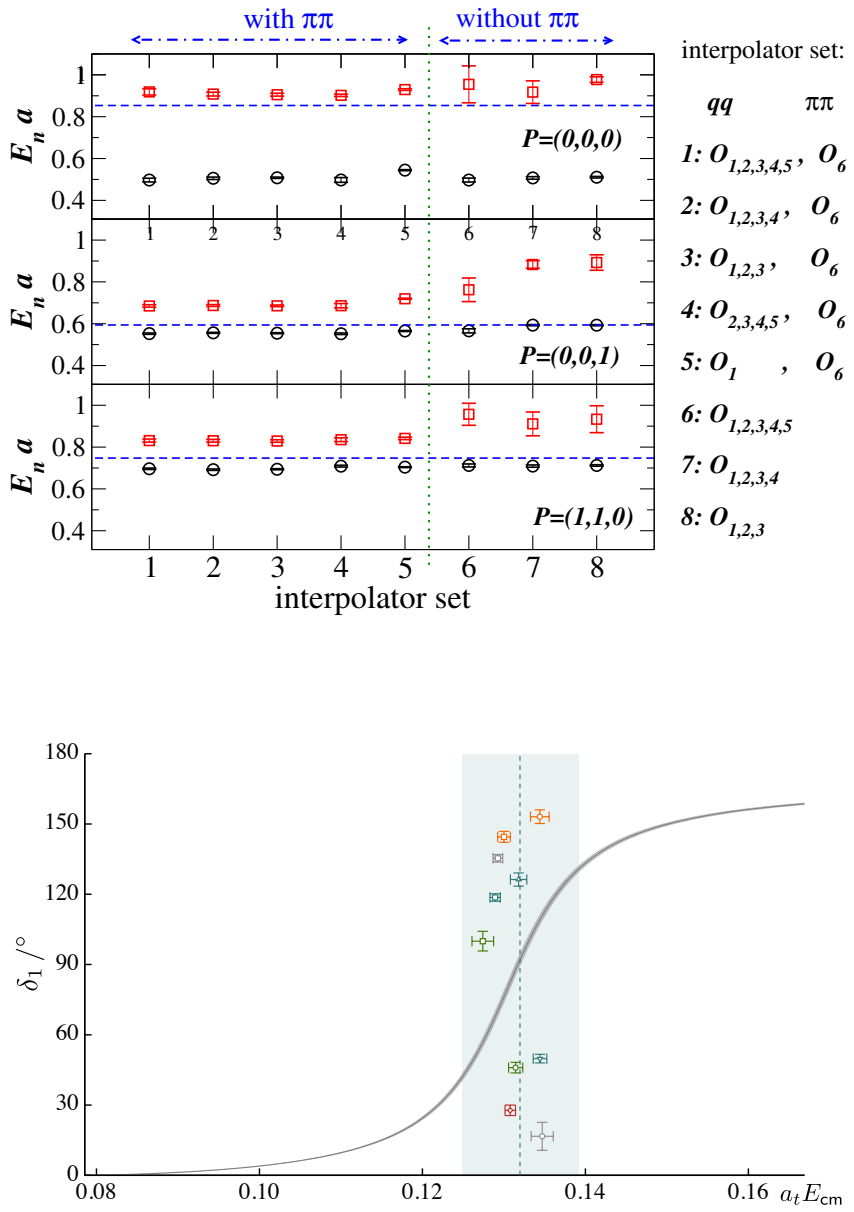

Figure 15. The lowest two energy levels from isospin-1 $\pi \pi$ scattering extracted using different sub-matrices (interpolators sets) of the full $16 \times 16$ correlation matrix. For details on the basis used please refer to [11].
Figure 16. Elastic phase shift points extracted using only single-hadron-like interpolators (data points) compared to the Breit-Wigner parameterization of the phase shift points in Figure 4. The vertical band indicates the Breit-Wigner mass and width. Figure from [15].

To illustrate this point further it is instructive to take a look at how the simulation results change when the full basis used in a given study is artificially truncated. This is illustrated in Figures 15 and 16. Figure 15 shows results for low-lying energy in three momentum frames from an early simulation of $\pi \pi$ scattering in the $\rho$ meson channel [11]. When the basis consisting of both quark-antiquark and meson-meson interpolating operators is reduced to contain just quark-antiquark operators, some of the excited-state energy levels become either ill-determined or display fake-plateaus, not representative of the physical spectrum. Figure 16 from [15] provides an illustration of the same problem: Using only the energy levels from single hadron (quark-antiquark) interpolators, the true phase-shift shown with the fit extracted from the full data set (curve) is missed and one would naively extract a much narrower resonance. 


\subsection{Baryon resonances in Meson-Baryon scattering}

Studies of baryon-meson scattering on the lattice are complicated by several aspects. For one, the signal to noise ratio at large Euclidean times is exponentially suppressed, leading to much noisier data. Furthermore the number of possible contractions is usually larger and the objects one calculates are more complex. Frames with total nonzero momentum lead to parity mixing in the lattice data, which makes the extraction of the spectrum even more challenging.

Consequently most studies of baryon spectroscopy to date extracted the energy levels from threequark interpolators only. In most cases multi-hadron levels are absent from the resulting spectra, and the cautionary remarks from Section 2.3 also apply to these studies. While showing some qualitative features of the physical spectrum, it is unclear to what extent such studies can be trusted. In this section, we therefore focus on exploratory studies including baryon-meson or other five-quark interpolating fields.

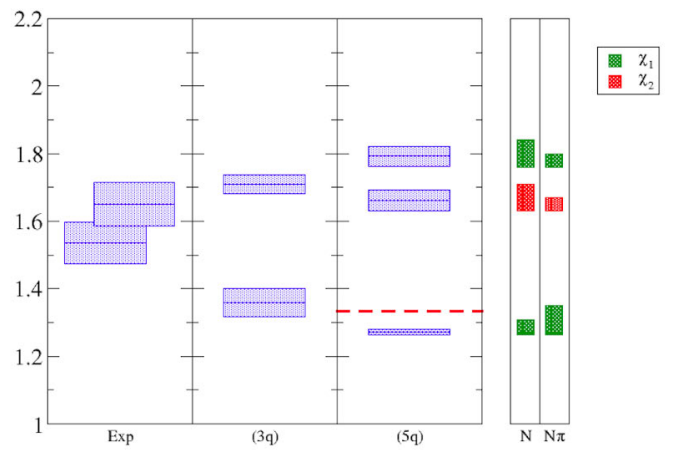

Figure 17. Results for the S-wave negative parity Nucleon spectrum. The left column shows the resonances observed in experiment shifted up by $\Delta E=130 \mathrm{MeV}$. The other columns show the lattice results from 3-quark interpolators alone (3q) and from the full basis consisting of 3-quark and Nucleon-pion interpolators. For a full explanation see [32].

A first study of low-lying negative parity nucleons has been performed by Lang and Verduci $[32,33]$. Figure 17 shows their results on a single volume with a pion mass of $266 \mathrm{MeV}$. Just like the meson studies discussed before, the spectrum using just single hadron interpolators is incomplete. Once meson-baryon interpolators are included, the low-lying energy spectrum can be extracted with reasonable precision. Future lattice QCD studies of negative parity baryon resonances will however require more volumes and/or the use of moving frames as well as additional scattering channels.

A more recent study by the Adelaide group [34] explored the use of local 5-quark interpolators for extracting the positive parity nucleon spectrum. Figure 18 shows the dependence of the energy levels observed when varying the interpolator basis. The complete basis consisted of 3-quark and local 5-quark interpolators with structures resembling $N \pi, N \sigma$, and $N a_{0}$ states. Unlike [33] this study does not result in energy levels close to the relevant thresholds and the spectrum should probably considered to be incomplete. The authors suggest that their results favor a scenario where the Roper resonance is a dynamically generated resonance.

\section{Summary and outlook}

Recent years have seen a lot of activity with regard to exploratory studies of resonances and bound states in meson-meson scattering. In the last two years the first coupled-channel simulations have appeared. At the same time calculations of meson-baryon energy levels are still scarce and a rigorous extraction of low-lying baryon resonances from such calculations is facing both conceptional and 


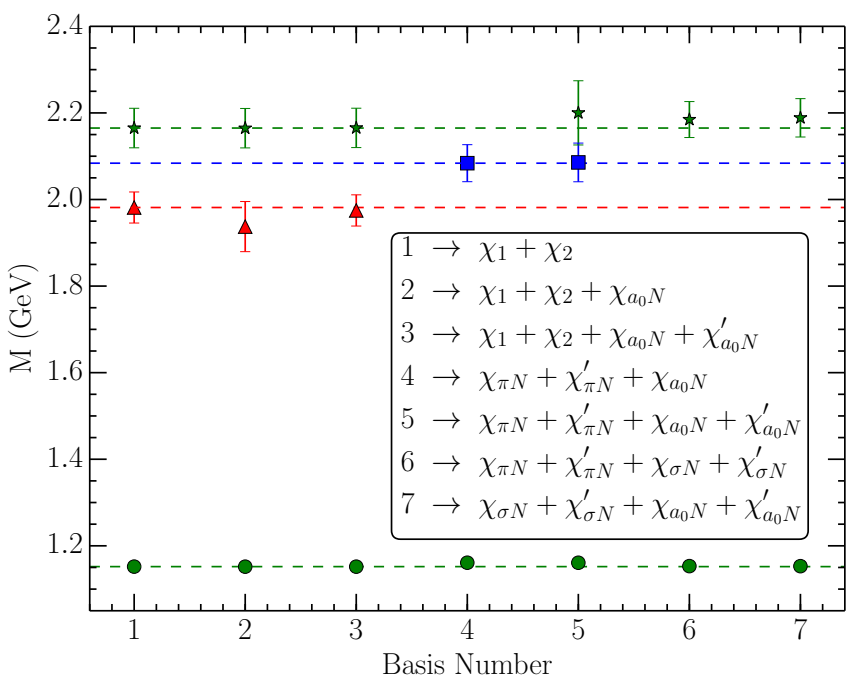

Figure 18. Low lying states from various sub-matrices of the full interpolator basis. $\chi_{1}$ and $\chi_{2}$ correspond to standard 3-quark nucleon interpolator, while $\chi_{a_{0} N}, \chi_{\pi N}$, and $\chi_{\sigma N}$ are local 5-quark interpolators with the structure of these meson-baryon combinations. Figure from [34].

computational obstacles. Since the talk these proceedings are based on, further results on the $\rho$ meson [35-37] and on baryon-meson scattering in the Roper channel [38] have appeared. Furthermore there are a number of results from meson-meson scattering involving heavy quarks which I could not cover. For a recent review of those results please refer to [39].

All of these calculations are currently of an exploratory nature, and future lattice studies will have to further address the systematic uncertainties, for example arising from discretization effects, exponential volume corrections, the pion mass dependence of observables, and, in some cases, neglected three-particle channels. In light of the many challenges one should recall that, unlike approaches based on models, the results of this programme are parameter free predictions of QCD.

\section{References}

[1] R.J. Dowdall, C.T.H. Davies, T.C. Hammant, R.R. Horgan, Phys. Rev. D86, 094510 (2012), 1207.5149

[2] S. Borsanyi et al., Science 347, 1452 (2015), 1406. 4088

[3] M. Lüscher, Commun. Math. Phys. 105, 153 (1986)

[4] M. Lüscher, Nucl. Phys. B354, 531 (1991)

[5] M. Lüscher, Nucl. Phys. B364, 237 (1991)

[6] R.A. Briceño, PoS LATTICE2014, 008 (2015), 1411.6944

[7] M.T. Hansen, PoS LATTICE2015, 008 (2016), 1511.04737

[8] C. Patrignani et al. (Particle Data Group), Chin. Phys. C40, 100001 (2016)

[9] S. Aoki et al. (CP-PACS), Phys. Rev. D76, 094506 (2007), 0708. 3705

[10] X. Feng, K. Jansen, D.B. Renner, Phys. Rev. D83, 094505 (2011), 1011.5288

[11] C.B. Lang, D. Mohler, S. Prelovsek, M. Vidmar, Phys. Rev. D84, 054503 (2011), [Erratum: Phys. Rev.D89,no.5,059903(2014)], 1105.5636

[12] S. Aoki et al. (CS), Phys. Rev. D84, 094505 (2011), 1106.5365

[13] C. Pelissier, A. Alexandru, Phys. Rev. D87, 014503 (2013), 1211.0092

[14] J.J. Dudek, R.G. Edwards, C.E. Thomas (Hadron Spectrum), Phys. Rev. D87, 034505 (2013), [Erratum: Phys. Rev.D90,no.9,099902(2014)], 1212.0830 
[15] D.J. Wilson, R.A. Briceno, J.J. Dudek, R.G. Edwards, C.E. Thomas, Phys. Rev. D92, 094502 (2015), 1507.02599

[16] G.S. Bali, S. Collins, A. Cox, G. Donald, M. Göckeler, C.B. Lang, A. Schäfer (RQCD), Phys. Rev. D93, 054509 (2016), 1512 .08678

[17] J. Bulava, B. Fahy, B. Hörz, K.J. Juge, C. Morningstar, C.H. Wong, Nucl. Phys. B910, 842 (2016), 1604.05593

[18] D. Guo, A. Alexandru, R. Molina, M. Döring, Phys. Rev. D94, 034501 (2016), 1605.03993

[19] B. Hu, R. Molina, M. Döring, A. Alexandru, Phys. Rev. Lett. 117, 122001 (2016), 1605.04823

[20] J. Bulava, B. Hörz, B. Fahy, K.J. Juge, C. Morningstar, C.H. Wong, PoS LATTICE2015, 069 (2016), 1511.02351

[21] H.B. Meyer, Phys. Rev. Lett. 107, 072002 (2011), 1105. 1892

[22] D. Bernecker, H.B. Meyer, Eur. Phys. J. A47, 148 (2011), 1107. 4388

[23] X. Feng, S. Aoki, S. Hashimoto, T. Kaneko, Phys. Rev. D91, 054504 (2015), 1412 . 6319

[24] D. Mohler, C.B. Lang, L. Leskovec, S. Prelovsek, R.M. Woloshyn, Phys. Rev. Lett. 111, 222001 (2013), 1308. 3175

[25] C.B. Lang, L. Leskovec, D. Mohler, S. Prelovsek, R.M. Woloshyn, Phys. Rev. D90, 034510 (2014), 1403.8103

[26] C.B. Lang, D. Mohler, S. Prelovsek, R.M. Woloshyn, Phys. Lett. B750, 17 (2015), 1501.01646

[27] J.J. Dudek, R.G. Edwards, C.E. Thomas, D.J. Wilson (Hadron Spectrum), Phys. Rev. Lett. 113, 182001 (2014), 1406.4158

[28] D.J. Wilson, J.J. Dudek, R.G. Edwards, C.E. Thomas, Phys. Rev. D91, 054008 (2015), 1411.2004

[29] J.J. Dudek, R.G. Edwards, D.J. Wilson (Hadron Spectrum), Phys. Rev. D93, 094506 (2016), 1602.05122

[30] C.B. Lang, L. Leskovec, D. Mohler, S. Prelovsek, Phys. Rev. D86, 054508 (2012), 1207. 3204

[31] S. Prelovsek, L. Leskovec, C.B. Lang, D. Mohler, Phys. Rev. D88, 054508 (2013), 1307.0736

[32] V. Verduci, C.B. Lang, PoS LATTICE2014, 121 (2014), 1412 . 0701

[33] C.B. Lang, V. Verduci, Phys. Rev. D87, 054502 (2013), 1212.5055

[34] A.L. Kiratidis, W. Kamleh, D.B. Leinweber, Z.W. Liu, F.M. Stokes, A.W. Thomas (2016), 1608.03051

[35] Z. Fu, L. Wang, Phys. Rev. D94, 034505 (2016), 1608.07478

[36] L. Leskovec, C. Alexandrou, G. Koutsou, S. Meinel, J.W. Negele, S. Paul, M. Petschlies, A. Pochinsky, G. Rendon, S. Syritsyn, A study of the radiative transition $\pi \pi \rightarrow$ $\pi \gamma^{*}$ with lattice $Q C D$, in Proceedings, 34th International Symposium on Lattice Field Theory (Lattice 2016): Southampton, UK, July 24-30, 2016 (2016), 1611.00282, http://inspirehep.net/record/1495431/files/arXiv: 1611.00282.pdf

[37] F. Erben, J. Green, D. Mohler, H. Wittig, Towards extracting the timelike pion form factor on CLS 2-flavour ensembles, in Proceedings, 34th International Symposium on Lattice Field Theory (Lattice 2016): Southampton, UK, July 24-30, 2016 (2016), 1611.06805, http://inspirehep.net/record/1499493/files/arXiv: 1611.06805.pdf

[38] C.B. Lang, L. Leskovec, M. Padmanath, S. Prelovsek (2016), 1610.01422

[39] S. Prelovsek, Lattice studies of charmonia and exotics, in 7th International Workshop on Charm Physics (Charm 2015) Detroit, MI, USA, May 18-22, 2015 (2015), 1508.07322, http://inspirehep.net/record/1390979/files/arXiv: 1508.07322.pdf 